\title{
The Impact of a Stabilization Exercise on Neck Pain: A Systematic Review and Meta-analysis
}

\author{
Binbin Wu ${ }^{1}$ Hongyan Yuan ${ }^{1}$ Deyu Geng ${ }^{1} \quad$ Liang Zhang $^{1} \quad$ Cheng Zhang ${ }^{1}$ \\ ${ }^{1}$ Department of Orthopedics, Qingdao Hospital of Traditional \\ Chinese Medicine (Qingdao Hiser Hospital), Shandong, China \\ J Neurol Surg A 2020;81:342-347.

\begin{abstract}
Address for correspondence Dr. Cheng Zhang, Department of Orthopedics, Qingdao Hospital of Traditional Chinese Medicine (Qingdao Hiser Hospital), 4 Renmin Road, Qingdao, Shandong, 325000, China (e-mail: zangcheng100@163.com).
\end{abstract}

\section{Abstract \\ Keywords \\ - stabilization exercise \\ - neck pain \\ - randomized controlled trials}

Introduction The efficacy of a stabilization exercise for the relief of neck pain remains controversial. We conducted a systematic review and meta-analysis to explore the effectiveness of a stabilization exercise on neck pain.

Methods We searched Embase, Web of Science, EBSCO Information Services, and the Cochrane Library databases through May 2019 for randomized controlled trials (RCTs) assessing the impact of a stabilization exercise on neck pain. This meta-analysis was performed using the random effects model.

Results Six RCTs are included in the meta-analysis. Compared with the control group of patients with neck pain, a stabilization exercise can significantly reduce pain scores at 4 to 6 weeks (mean difference [MD]: $-2.41 ; 95 \%$ confidence interval [CI], -4.46 to $-0.35 ; p=0.02$ ), Neck Disability Index [NDI] at 10 to 12 weeks (MD:- $6.75 ; 95 \%$ $\mathrm{Cl},-11.71$ to $-1.79 ; p=0.008$ ), and depression scale at 4 to 6 weeks (MD: $-4.65 ; 95 \%$ $\mathrm{Cl},-7.00$ to $-2.31 ; p=0.02)$, but it has no obvious impact on pain scores at 10 to 12 weeks (MD: $-1.07 ; 95 \% \mathrm{Cl},-3.42$ to $1.28 ; p=0.37$ ) or at 6 months (MD: $-1.02 ; 95 \%$ $\mathrm{Cl},-3.43$ to $1.39 ; p=0.41)$.

Conclusions A stabilization exercise can provide some benefits to control neck pain.

\section{Introduction}

Neck pain is reported to rank as the second most prevalent musculoskeletal disorder. ${ }^{1-3}$ It is estimated that $\sim 67 \%$ of people may experience neck pain during their lifetime., ${ }^{4,5}$ Neck pain results in a reduced quality of daily life and has a considerable economic impact on health care systems. Its diagnosis and treatment are still challenging because the anatomical source and cause of neck pain remains elusive in most cases. ${ }^{6}$

Neck pain may be associated with a reduction in the strength and endurance capacity of the cervical muscles. ${ }^{7,8}$ For instance, the strength of deep and anterior cervical flexors in the cervical spine is reduced in patients with neck pain. ${ }^{9}$ Exercise can possibly alleviate neck pain because of its ability to gain muscle strength, flexibility, and endur-

received

March 12, 2019

accepted after revision

March 12, 2019

published online

March 6, 2020

ance, as well as restore injured tissues. ${ }^{10}$ Stabilization exercises serve as an important approach to alleviate back and pelvic pain, ${ }^{11-13}$ and they have the ability to achieve a stable injury-free state for the cervical spine. ${ }^{14,15}$

Several studies reported on the treatment efficacy of stabilization exercises on neck pain, but the results are conflicting. ${ }^{4} 16-18$ This meta-analysis of randomized controlled trials (RCTs) aimed to investigate the treatment efficacy and function of stabilization exercises versus general exercises for patients with neck pain.

\section{Materials and Methods}

This systematic review and meta-analysis were performed based on the guidance of the Preferred Reporting Items for Systematic Reviews and Meta-analysis statement and

(c) 2020 Georg Thieme Verlag KG Stuttgart · New York
DOI https://doi.org/ 10.1055/s-0039-3400953. ISSN 2193-6315. 
Cochrane Handbook for Systematic Reviews of Interventions. $^{19,20}$ No ethical approval and patient consent were required because all analyses were based on previously published studies.

\section{Literature Search and Selection Criteria}

We systematically searched several databases including Embase, Web of Science, EBSCO Information Services, and the Cochrane Library from inception to May 2019 with the key words stabilization exercise and neck pain. The reference lists of retrieved studies and relevant reviews were also hand-searched, and the search process as described was performed repeatedly to include additional eligible studies.

The inclusion criteria are presented as follows: (1) study design is RCT, (2) patients are diagnosed with neck pain, and (3) intervention treatments are stabilization exercises versus general exercise. Patients with cervical radiculopathy were excluded.

\section{Data Extraction and Outcome Measures}

Some baseline information was extracted from the original studies including first author, number of patients, age, female, body mass index (BMI), and detailed methods in the two groups. Data were extracted independently by two investigators, and discrepancies were resolved by consensus. We contacted the corresponding author to obtain data when necessary.

The primary outcomes are pain scores at 4 to 6 weeks and 10 to 12 weeks. Secondary outcomes are pain scores at 6 months, Neck Disability Index (NDI) at 10 to 12 weeks, and depression scale at 4 to 6 weeks.

\section{Quality Assessment in Individual Studies}

The methodological quality of each RCT was assessed by the Jadad Scale that consists of three evaluation elements: randomization ( $0-2$ points), blinding ( $0-2$ points), and dropouts and withdrawals ( $0-1$ points). ${ }^{21}$ One point is allocated to each element if they have been conducted and mentioned appropriately in the original article. The Jadad Scale scores vary from 0 to 5 points. An article with a Jadad score $\leq 2$ is considered low quality; it is considered high quality with a Jadad score $\geq 3$. $^{22}$

\section{Statistical Analysis}

We assessed mean differences (MDs) with 95\% confidence intervals (CIs) for continuous outcomes (pain scores at 4-6 weeks, 10-12 weeks, and 6 months; NDI at 10-12 weeks; and depression scale at 4-6 weeks). Heterogeneity was evaluated using the $\mathrm{I}^{2}$ statistic with $\mathrm{I}^{2}>50 \%$ indicating significant heterogeneity. ${ }^{23}$ The random effects model was used for all metaanalyses. We searched for potential sources of heterogeneity for significant heterogeneity. Sensitivity analysis was performed to detect the influence of a single study on the overall estimate via omitting one study in turn or performing the subgroup analysis. Owing to the limited number $(<10)$ of the included studies, publication bias was not assessed. Results were considered statistically significant for $p<0.05$. All statistical analyses were performed using RevMan, v.5.3 (The Cochrane Collaboration, Software Update, Oxford, United Kingdom).

\section{Results}

\section{Literature Search, Study Characteristics, and Quality Assessment}

A detailed flowchart of the search and selection results is shown in -Fig. 1. A total of 469 potentially relevant articles were identified initially. Six RCTs that ultimately met our inclusion criteria were included in the meta-analysis., 16-18,24,25

-Table 1 presents the main characteristics of the six included RCTs. The six studies were published between 2003 and 2019, and the total sample size was 395. Five included RCTs involved chronic neck pain, 4,16,18,24,25 and the remaining RCT involved neck pain caused by posttraumatic stress disorder. ${ }^{17}$

Among the six RCTs, four included RCTs that reported pain scores at 4 to 6 weeks, ${ }^{16-18,25}$ two included RCTs that reported pain scores at 10 to 12 weeks, ${ }^{4,25}$ two included RCTs that reported pain scores at 6 months, ${ }^{18,25}$ two included RCTs that reported the NDI at 10 to 12 weeks, ${ }^{4,25}$ and two included RCTs that reported the depression scale at 4 to 6 weeks. ${ }^{17,25}$ Jadad scores of the six included studies varied from 3 to 5 points, and all six studies were considered high quality, according to the quality assessment.

\section{Primary Outcomes: Pain Scores at 4 to 6 Weeks and 10 to 12 Weeks}

The random effects model was used for the analysis of pain scores at 4 to 6 weeks and 10 to 12 weeks. Compared with the control group of patients with neck pain, stabilization exercises were associated with significantly reduced pain scores at 4 to 6 weeks (MD: $-2.41 ; 95 \% \mathrm{CI},-4.46$ to $-0.35 ; p=0.02$ ) with significant heterogeneity among the studies ( $\mathrm{I}^{2}: 94 \%$; heterogeneity $p<0.00001$; - Fig. 2) but showed no notable impact on pain scores at 10 to 12 weeks (MD: $-1.07 ; 95 \% \mathrm{CI},-3.42$ to $1.28 ; p=0.37$ ) with significant heterogeneity among the studies $\left(I^{2}: 91 \%\right.$; heterogeneity $p=0.0009 ;$ - Fig. 3$)$.

\section{Sensitivity Analysis}

Significant heterogeneity was observed among the included studies for the primary outcomes, and significant heterogeneity remained when performing sensitivity analysis by omitting one study in turn.

\section{Secondary Outcomes}

Stabilization exercises were found to have no substantial influence on pain scores at 6 months compared with the control group for neck pain (MD: $-1.02 ; 95 \% \mathrm{Cl},-3.43$ to 1.39 ; $p=0.41$; - Fig. 4), but did result in a reduction in NDI at 10 to 12 weeks (MD: $-6.75 ; 95 \% \mathrm{Cl},-11.71$ to -1.79 ; $p=0.008$; - Fig. 5) and depression scale at 4 to 6 weeks (MD: $-4.65 ; 95 \% \mathrm{CI},-7.00$ to $-2.31 ; p=0.02$; - Fig. 6).

\section{Discussion}

Altered motor control is confirmed in chronic neck pain. 6,26,27 Different aspects are associated with altered motor control and include co-contraction of agonistic muscles, more activity for superficial flexors and extensors, 


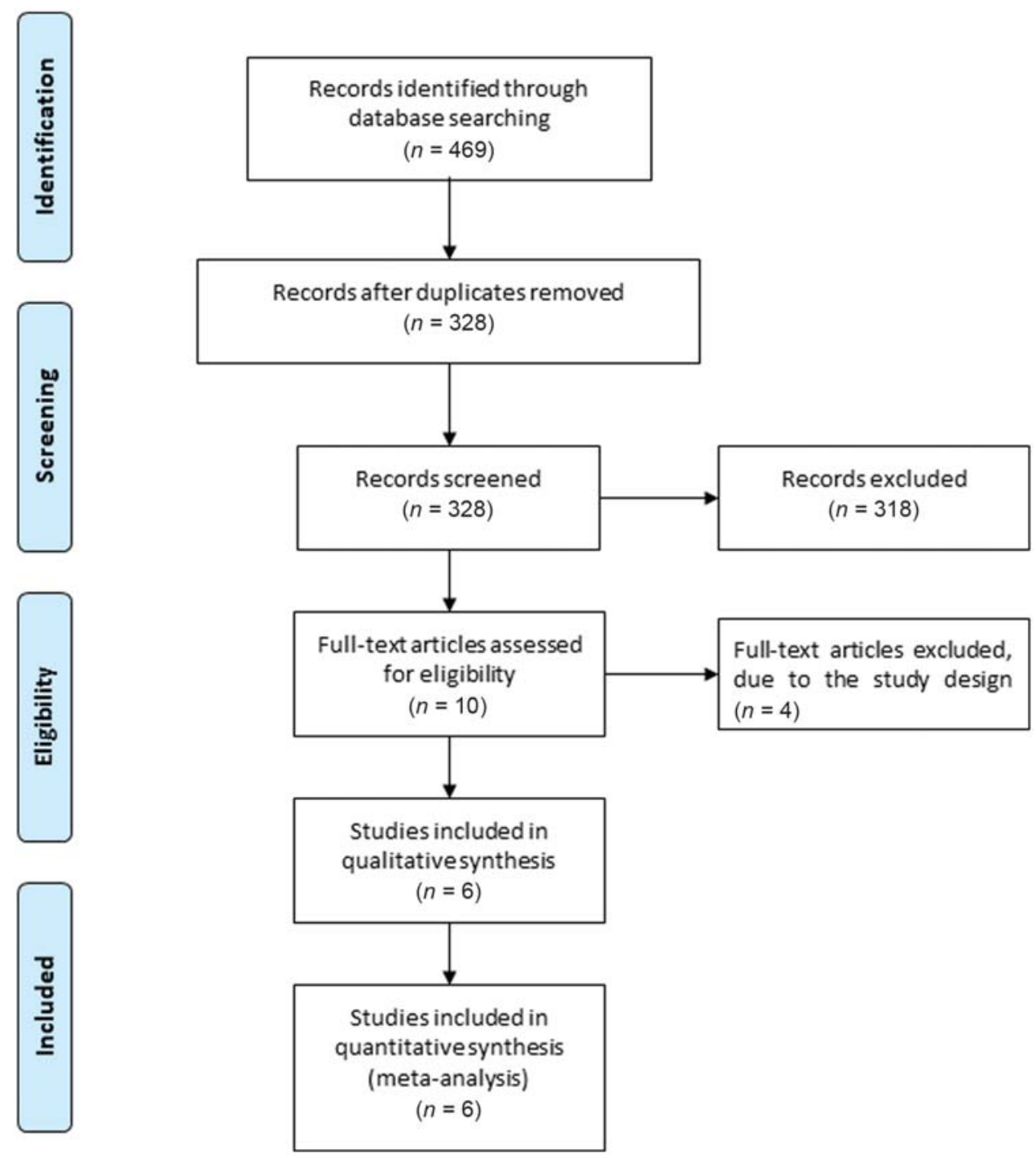

Fig. 1 Flow diagram of study search and selection process.

delayed onset time for neck muscles, and weakness of deep muscles. ${ }^{6,28-30}$ The muscles are found to provide $\sim 80 \%$ of spinal stabilization. ${ }^{31}$ Deep and superficial muscles have different roles for cervical stabilization. Deep muscles are segmental stabilizers and provide stabilization between segments that is the basis for participation of the superficial muscles. ${ }^{6}$

Patients with neck pain have weak deep cervical flexors that may result in increased activity level of the superficial flexor muscles. ${ }^{6}$ These patients have reduced deep muscle activity and increased superficial muscle activity during cognitive tasks and functional activities. Increased activity of superficial flexors during isometric contraction is also reported to cause neck pain. ${ }^{28}$ High co-contraction of a weak sternocleidomastoid and the anterior scalene can lead to neck pain and disability. ${ }^{32}$ A specific exercise program should be designed to target the deep flexors first and then the superficial ones.

Cervical stabilization exercises can improve neck pain and cervical muscle performance in patients with cervicogenic headache. ${ }^{12}$ When compared with isometric and stretching exercises, stabilization exercises may be more effective in improving disability and pain control for patients with neck pain. ${ }^{25}$ Our meta-analysis concluded that stabilization exercises can substantially reduce pain scores at 4 to 6 weeks, NDI at 10 to 12 weeks, and depression scale at 4 to 6 weeks for neck pain patients, but they show no obvious influence on pain scores at 10 to 12 weeks or 6 months. In one included RCT involving the influence of exercise programs on neck pain, the results found significantly increased deep flexor muscle endurance for stabilization exercise and also increased endurance for a group of routine exercises. ${ }^{4}$

Significant heterogeneity is observed when omitting one study in turn for the sensitivity analysis. Several factors may account for this heterogeneity, and they include different causes (e.g., chronic neck pain and posttraumatic stress disorder) of neck pain, various combinations with stabilization exercises (e.g., isometric neck strengthening and physical therapy), and duration of administration (ranging from 6 weeks to 12 months).

This study has several possible limitations. First, only six RCTs were included in this meta-analysis, and five of them 


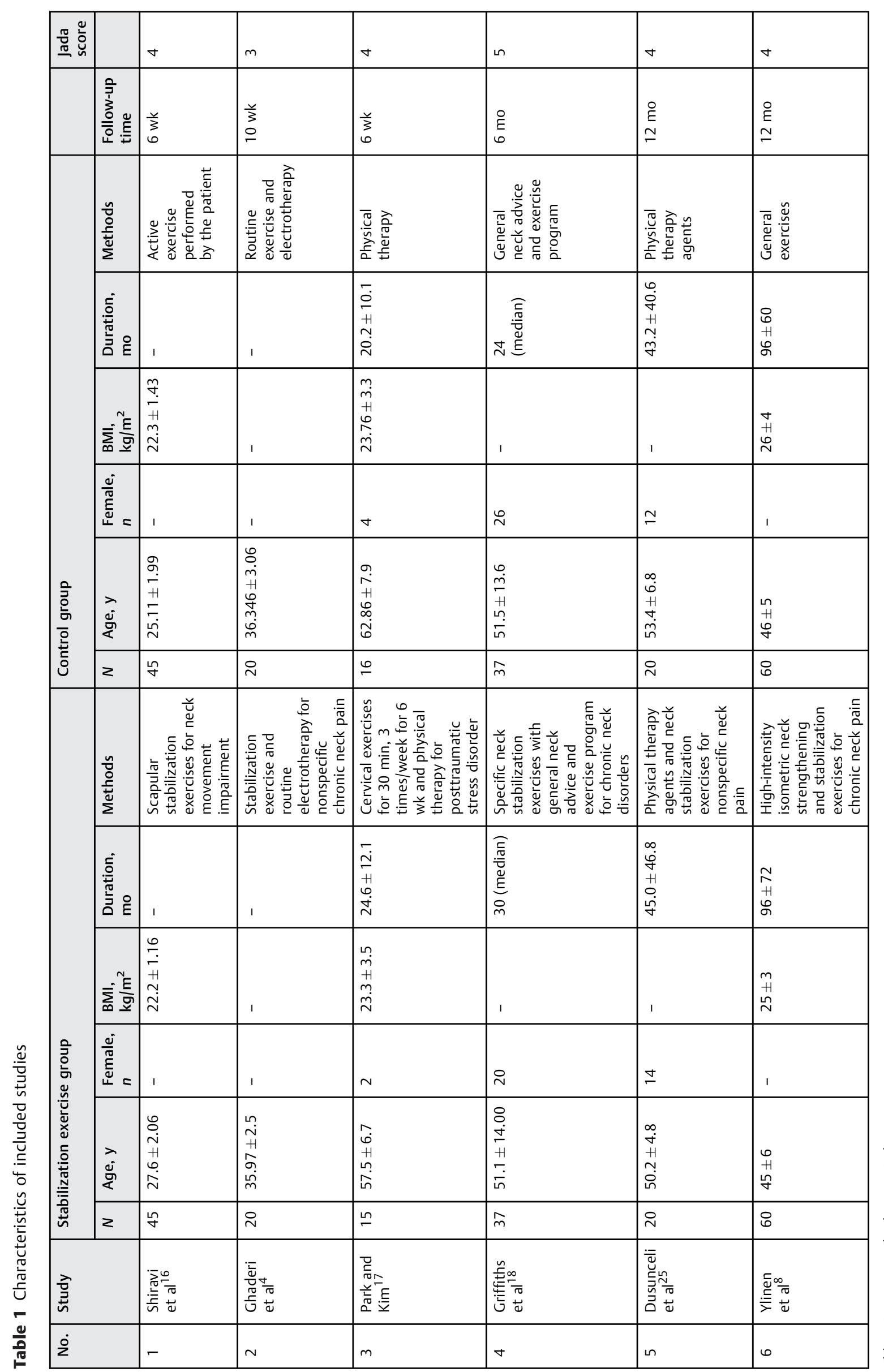




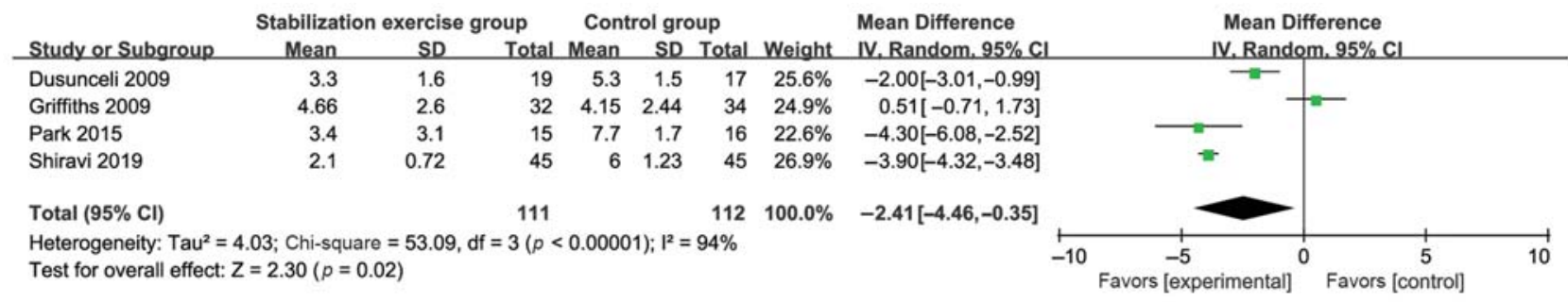

Fig. 2 Forest plot for meta-analysis of pain scores at 4 to 6 weeks. Cl, confidence interval; IV, inverse variance; SD, standard deviation.

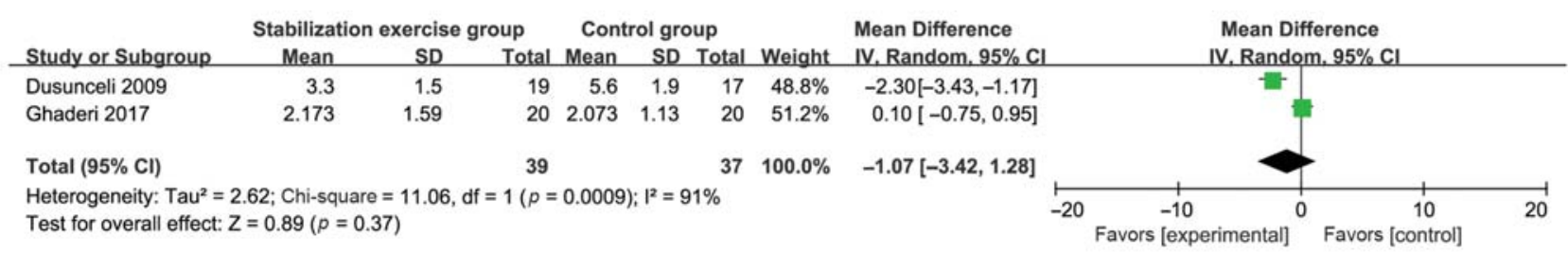

Fig. 3 Forest plot for meta-analysis of pain scores at 10 to 12 weeks. Cl, confidence interval; IV, inverse variance; SD, standard deviation.

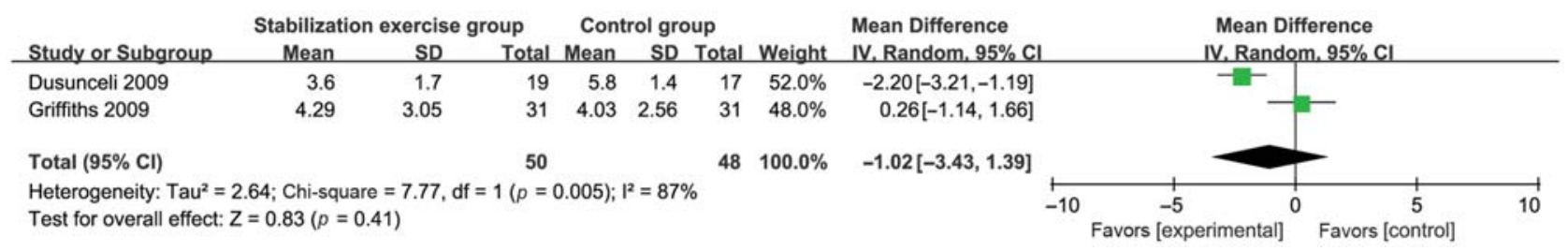

Fig. 4 Forest plot for meta-analysis of pain scores at 6 months. Cl, confidence interval; IV, inverse variance; SD, standard deviation.

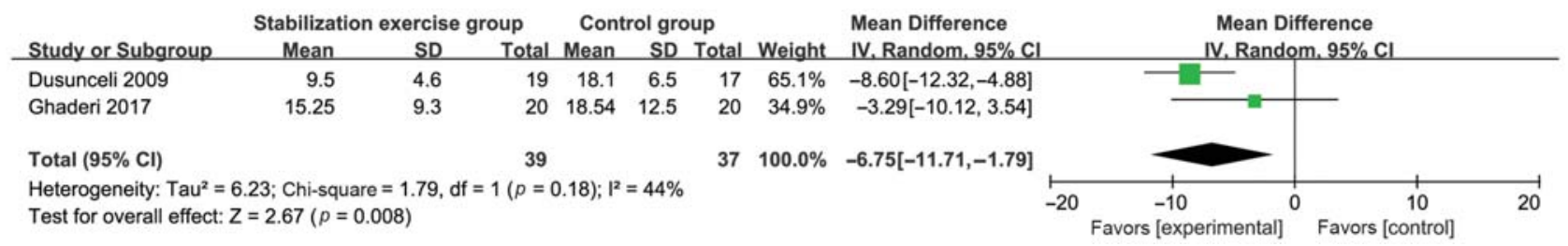

Fig. 5 Forest plot for meta-analysis of Neck Disability Index (NDI) at 10 to 12 weeks. CI, confidence interval; IV, inverse variance; SD, standard deviation.

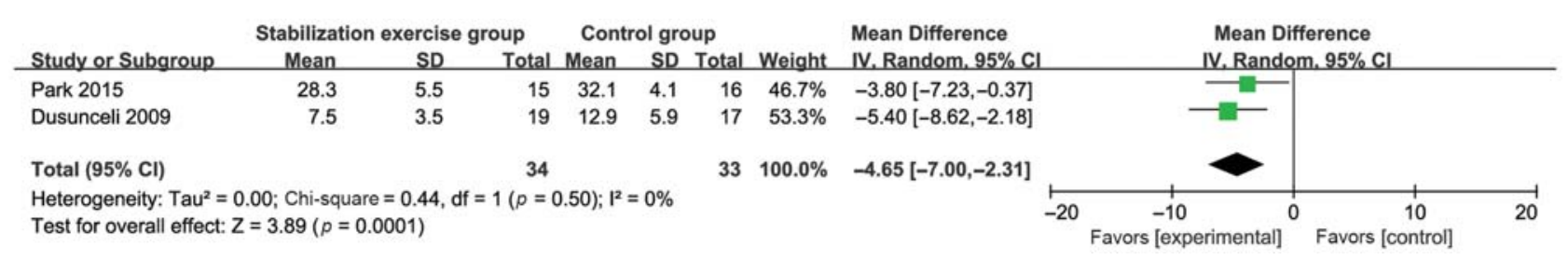

Fig. 6 Forest plot for meta-analysis of depression scale at 4 to 6 weeks. Cl, confidence interval; IV, inverse variance; SD, standard deviation.

had a small sample size $(n<100)$. Overestimation of the treatment effect is more likely in smaller trials compared with larger samples. Next, significant heterogeneity was observed in this meta-analysis and may have been caused by different methods and duration of stabilization exercises, as well as sex. Finally, it was not feasible to perform a metaanalysis of some important outcomes such as endurance or the SF-36 Short Form. In conclusion, stabilization exercises can provide some benefit for pain relief and cervical function for patients with neck pain.
Conflicts of Interest

None declared.

\section{References}

1 Côté P, Cassidy JD, Carroll L. The Saskatchewan Health and Back Pain Survey. The prevalence of neck pain and related disability in Saskatchewan adults. Spine 1998;23(15):1689-1698

2 Genebra CVDS, Maciel NM, Bento TPF, Simeão SFAP, Vitta A. Prevalence and factors associated with neck pain: a populationbased study. Braz J Phys Ther 2017;21(04):274-280 
3 Ye S, Jing Q Wei C, Lu J. Risk factors of non-specific neck pain and low back pain in computer-using office workers in China: a crosssectional study. BMJ Open 2017;7(04):e014914

4 Ghaderi F, Jafarabadi MA, Javanshir K. The clinical and EMG assessment of the effects of stabilization exercise on nonspecific chronic neck pain: a randomized controlled trial. J Back Musculoskeletal Rehabil 2017;30(02):211-219

5 Fougeront N, Fleiter B. Temporomandibular disorder and comorbid neck pain: facts and hypotheses regarding pain-induced and rehabilitation-induced motor activity changes. Can J Physiol Pharmacol 2018;96(11):1051-1059

6 Falla D. Unravelling the complexity of muscle impairment in chronic neck pain. Man Ther 2004;9(03):125-133

7 Chiu TT, Sing KL. Evaluation of cervical range of motion and isometric neck muscle strength: reliability and validity. Clin Rehabil 2002;16(08):851-858

8 Ylinen J, Salo P, Nykänen M, Kautiainen H, Häkkinen A. Decreased isometric neck strength in women with chronic neck pain and the repeatability of neck strength measurements. Arch Phys Med Rehabil 2004;85(08):1303-1308

9 Gogia PP, Sabbahi MA. Electromyographic analysis of neck muscle fatigue in patients with osteoarthritis of the cervical spine. Spine 1994;19(05):502-506

10 Wolsko PM, Eisenberg DM, Davis RB, Kessler R, Phillips RS. Patterns and perceptions of care for treatment of back and neck pain: results of a national survey. Spine 2003;28(03):292-297; discussion 298

11 Kose G, Hepguler S, Atamaz F, Oder G. A comparison of four disability scales for Turkish patients with neck pain.J Rehabil Med 2007;39(05):358-362

12 Jull G, Trott P, Potter $\mathrm{H}$, et al. A randomized controlled trial of exercise and manipulative therapy for cervicogenic headache. Spine 2002;27(17):1835-1843; discussion 1843

13 Jordan A, Mehlsen J, Ostergaard K. A comparison of physical characteristics between patients seeking treatment for neck pain and age-matched healthy people. J Manipulative Physiol Ther 1997;20(07):468-475

14 Monticone M, Barbarino A, Testi C, Arzano S, Moschi A, Negrini S. Symptomatic efficacy of stabilizing treatment versus laser therapy for sub-acute low back pain with positive tests for sacroiliac dysfunction: a randomised clinical controlled trial with 1 year follow-up. Eura Medicophys 2004;40(04):263-268

15 Arokoski JP, Valta T, Airaksinen O, Kankaanpää M. Back and abdominal muscle function during stabilization exercises. Arch Phys Med Rehabil 2001;82(08):1089-1098

16 Shiravi S, Letafatkar A, Bertozzi L, Pillastrini P, Khaleghi Tazji M. Efficacy of abdominal control feedback and scapula stabilization exercises in participants with forward head, round shoulder postures and neck movement impairment. Sports Health 2019; 11(03):272-279
17 Park SD, Kim SY. Clinical feasibility of cervical exercise to improve neck pain, body function, and psychosocial factors in patients with post-traumatic stress disorder: a randomized controlled trial. J Phys Ther Sci 2015;27(05):1369-1372

18 Griffiths C, Dziedzic K, Waterfield J, Sim J. Effectiveness of specific neck stabilization exercises or a general neck exercise program for chronic neck disorders: a randomized controlled trial. J Rheumatol 2009;36(02):390-397

19 Moher D, Liberati A, Tetzlaff J, Altman DG; PRISMA Group. Preferred reporting items for systematic reviews and meta-analyses: the PRISMA statement. BMJ 2009;339:b2535

20 Higgins JPT, Green S. Cochrane Handbook for Systematic Reviews of Interventions [version 5.1.0]. Chichester, UK: John Wiley; 2011

21 Jadad AR, Moore RA, Carroll D, et al. Assessing the quality of reports of randomized clinical trials: is blinding necessary? Control Clin Trials 1996;17(01):1-12

22 Kjaergard LL, Villumsen J, Gluud C. Reported methodologic quality and discrepancies between large and small randomized trials in meta-analyses. Ann Intern Med 2001;135(11):982-989

23 Higgins JP, Thompson SG. Quantifying heterogeneity in a metaanalysis. Stat Med 2002;21(11):1539-1558

24 Ylinen J, Takala EP, Nykänen M, et al. Active neck muscle training in the treatment of chronic neck pain in women: a randomized controlled trial. JAMA 2003;289(19):2509-2516

25 Dusunceli Y, Ozturk C, Atamaz F, Hepguler S, Durmaz B. Efficacy of neck stabilization exercises for neck pain: a randomized controlled study. J Rehabil Med 2009;41(08):626-631

26 Woodhouse A, Vasseljen O. Altered motor control patterns in whiplash and chronic neck pain. BMC Musculoskelet Disord 2008; 9:90

27 De Pauw R, Coppieters I, Caeyenberghs K, et al. Associations between brain morphology and motor performance in chronic neck pain: a whole-brain surface-based morphometry approach. Hum Brain Mapp 2019

28 Falla D, Jull G, Hodges PW. Feedforward activity of the cervical flexor muscles during voluntary arm movements is delayed in chronic neck pain. Exp Brain Res 2004;157(01):43-48

29 Falla D, Lindstrøm R, Rechter L, Farina D. Effect of pain on the modulation in discharge rate of sternocleidomastoid motor units with force direction. Clin Neurophysiol 2010;121(05):744-753

30 Falla D, Rainoldi A, Merletti R, Jull G. Spatio-temporal evaluation of neck muscle activation during postural perturbations in healthy subjects. J Electromyogr Kinesiol 2004;14(04):463-474

31 Hides JA, Richardson CA, Jull GA. Multifidus muscle recovery is not automatic after resolution of acute, first-episode low back pain. Spine 1996;21(23):2763-2769

32 Lindstrøm R, Schomacher J, Farina D, Rechter L, Falla D. Association between neck muscle coactivation, pain, and strength in women with neck pain. Man Ther 2011;16(01):80-86 